\title{
Picture-word differences in decision latency: An analysis of single and dual memory models
}

\author{
JAMES W. PELLEGRINO, RICHARD R. ROSINSKI, HARRY L. CHIESI, \\ and ALEXANDER SIEGEL \\ University of Pittsburgh, Pittsburgh, Pennsylvania 15260
}

\begin{abstract}
Semantic and perceptual size decision times for pictorial and verbal material were analyzed in the context of a unitary memory model and several dual memory models. Experiment 1 involved a same-different categorical judgment task. The results showed that picture-picture response latencies were $185 \mathrm{msec}$ faster than the corresponding word-word latencies, and word-picture and picture-word latencies equaled the mean of these two extremes. Similarity of subcategory for "same" judgments led to faster decision latency for all presentation conditions. Additionally, a linear relationship was found between picture-picture and word-word latencies for individual item pairs. Experiment 2 involved a comparison of pictures and words across a categorical judgment and a size judgment task. Pictures produced faster decision latencies in both tasks, and the latency difference between pictures and words was comparable across tasks. These data fit the predictions of a unitary memory model. Several variants of a dual memory model are rejected and those which fit the data require assumptions about storage and/or transfer time values which result in a functional regression to the unitary memory model.
\end{abstract}

Research in human memory has identified a series of temporally defined levels or stages of processing generally denoted as sensory, short-term, and long-term memory. These stages have been shown to be affected both by the input modality of the stimuli (i.e., visual or auditory) and the type of stimulus material presented (i.e., pictures or words) (e.g., Penney, 1975). These modality and materials differences are, for the most part, independent of the question whether the processing of input involves qualitatively different stages (e.g., Wickelgren, 1973) or discrete points along a general continuum of processing (e.g., Craik \& Lockhart, 1972).

At the initial sensory processing level, there is strong support for separate visual (iconic) (Sperling, 1960) and acoustic (echoic) stores (e.g., Crowder \& Morton, 1969; Neisser, 1967). Other studies of short-term retention have demonstrated the existence of corresponding visual and acoustic short-term stores (e.g., Kroll, Parkinson, \& Parks, 1972; Pellegrino, Siegel, \& Dhawan, 1975, 1976).

This research was supported in part by funds provided by the Learning Research and Development Center, University of Pittsburgh, which is funded in part by the National Institute of Education, U. S. Department of Health, Education, and Welfare. The authors would like to express their appreciation to Micki Chi, Rob Kail, Alan Lesgold, Charles Perfetti, and James Voss for their helpful discussions of the present research. Special thanks go to Susan Goldman, whose insightful and constant criticisms greatly improved this paper. Reprint requests should be sent to James W. Pellegrino, Learning Research and Development Center, University of Pittsburgh, Pittsburgh, Pennsylvania 15260.
Long-term memory represents the third temporal level at which modality and materials differences have been observed. A large number of studies have shown a consistent superiority of pictorial relative to verbal input in a variety of standard long-term retention tasks (cf. Paivio, 1971). Paivio (1971) has argued that such results are consistent with a dual-coding hypothesis whereby pictorial stimuli generate simultaneous verbal and nonverbal long-term memory codes. However, since it is generally assumed that long-term retention depends upon item encoding in terms of the attributes of semantic or permanent memory (e.g., Atkinson \& Shiffrin, 1968; Craik \& Lockhart, 1972), the question remains as to the locus of these proposed dual memory codes. It is possible that the verbal and nonverbal codes are (1) components of a unitary longterm, permanent, or semantic memory system, or (2) components of two separate permanent memory systems. Another way to approach this issue is to ask whether the separate visual and acoustic sensory and short-term processing systems are access routes to corresponding different permanent memory systems or different access routes to a unitary abstract (multimodal) permanent memory system.

Paivio $(1971,1974,1975)$ has proposed a model of memory in which he postulates the existence of separate but interconnected verbal and nonverbal knowledge or symbolic systems. The model as represented in Figure 1 assumes that pictures have direct access to the nonverbal symbolic system while words have direct access to the verbal symbolic system. Another assumption of the model is that of transfer asymmetry, whereby transfer from the nonverbal to 


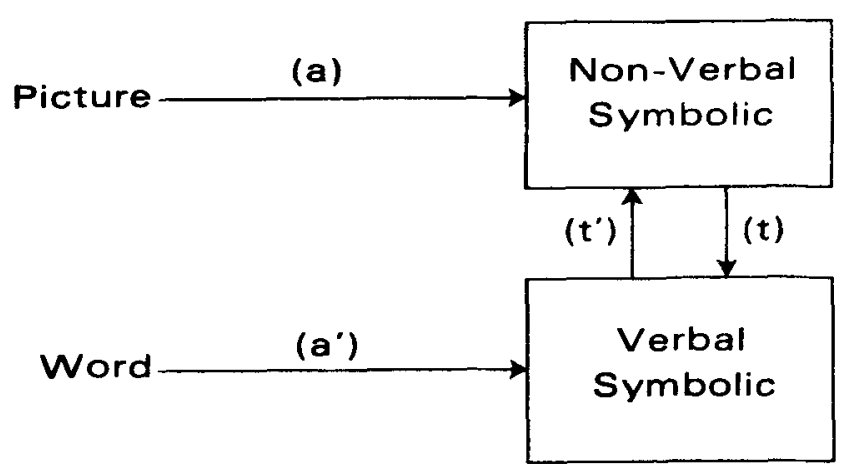

Figure 1. Simple representation of dual memory model with separate symbolic systems and access routes for pictorial and verbal input.

the verbal symbolic system is more rapid than the converse. Thus, the model as represented in Figure 1 typically assumes equal access times for both systems $\left(a=a^{\prime}\right)$ but an inequality in transfer times $\left(t<t^{\prime}\right)$. Therefore, because of the differential availability and ease of access to two long-term memory representations, long-term retention of pictures is superior to that of concrete words, which in turn is superior to that of abstract words. While Paivio's (1971, 1974, 1975) dual-coding model of memory is capable of accounting for differential long-term retention of pictures and words, it is not without its critics (e.g., Anderson \& Bower, 1973; Pylyshyn, 1973).

An alternative to the dual memory model is one which assumes a single abstract (or multimodal) permanent knowledge system (e.g., Anderson \& Bower, 1973; Chase \& Clark, 1972). This system is built on the basis of accumulated and organized perceptual experience and has multiple access routes, one of which involves verbal or linguistic processing. Concepts in this knowledge system would have multimodal attributes related to the various types of perceptual experience. Such a model is also congruent with the general position that memory involves a processing continuum where retention of input depends upon the depth, breadth, or levels of processing and the relation of such processing to the information in the permanent semantic store (Craik \& Lockhart, 1972).

The question of access to single or multiple permanent memory systems cannot be resolved by recourse to the presently available research on shortterm or long-term retention. The information provided is insufficient and the arguments in favor of the unitary memory model rest on logical rather than empirical grounds. The problem of comparing the two theoretical positions has been explicitly noted by Anderson and Bower (1973): "It is proven difficult to achieve any clear-cut differentiation and testing among these several points of view. Part of the problem of distinguishing the hypotheses is that the denotations of the 'verbal' and 'imagery' systems change in the theoretician's hands according to the explanatory demands of the situation. Imagery refers not only to our detailed memory of pictures and melodies, but also to vague, schematic memory for such stimuli, as well as to memory for the meaning of a sentence. On the other hand, the verbal medium is taken variously not only to denote words as acoustical stimuli, but also to denote semantic interpretations and rich, meaningful relationships among concepts. The terminology in this nether world has just become exceedingly imprecise" (pp. 452-453).

One method which may help to isolate differences between single and dual memory models with respect to storage assumptions and processing parameters is the decision latency task. Paivio (1974) has examined reaction time differences between pictures and words in a relative size comparison task. In this task, picture and word pairs were presented visually and the subject was required to select the item which had the larger real world referent (e.g., mouse vs. elephant). Paivio found that decision times for picture pairs were approximately $187 \mathrm{msec}$ faster than for word pairs. He argued that this was consistent with a dual memory position which postulates that the nonverbal symbolic system is organized in terms of perceptual attributes. The size judgment or comparison could only be mediated by information in the nonverbal symbolic system. Therefore, the word pairs were slower because of the necessary time required to transfer information from the verbal to the nonverbal symbolic system.

The argument that pictures have more direct access to the nonverbal system than words was also used by Paivio (1974) to predict and explain another set of results. In some of the pairs the larger real world referent (e.g., elephant) was presented so that it was physically smaller than the conceptually smaller referent (e.g., mouse). This was done in both the picture and word pairs.

"The clear prediction here was that, if the size comparisons involve the visual system, there should be a conflict between the tendencies to respond to the larger object in the picture and the larger one in memory when the pictured size relation is incongruent with the conceptual size relation. Thus the reaction time should be slower for the incongruous than the congruous picture condition. This effect should not occur for words differing in printed size, or at least the effect should be smaller, because the words must first be read before the images can be aroused and compared.... As predicted, the reaction times were slower for the incongruent than the congruent condition, and this difference was significantly larger for pictures than for words" (Paivio, 1974, p. 13).

While Paivio's (1974) data are consistent with a dual memory model, they are also consistent with a unitary memory model which assumes that pictures have faster access than words to a single permanent memory store. The difference in access time could be 
due to an acoustic-phonemic decoding process for words prior to higher level processing. Such a decoding process should not be affected by print size or differences in print size for the presented words. Furthermore, the size differential in word pairs was less than in picture pairs. Paivio's (1974) major argument against the unitary memory model was based on the difficulty of representing and comparing information from a perceptual continuum within a propositional format.

The ambiguity in interpreting Paivio's (1974) decision latency data reflects the need to develop more precisely stated models that lead to clear differences in predictions for different types of decision or processing tasks. The purpose of the present research was to develop models representing two different types of stored knowledge and to apply them to such decision latency tasks. The first experiment was designed to compare assumptions and predictions of single and dual memory models in a decision latency task based upon a more abstract and dichotomous dimension. The specific task was judging the semantic category identity of pairs of items presented pictorially and verbally. The general procedure was similar to one used by Rader (Note 1) and involved the simultaneous presentation of two pictures, the corresponding pair of words, or the corresponding picture-word or word-picture pair. The subjects were required to make same-different semantic category decisions in a reaction time task. Latency differences between pictures and words in this task can be related to Paivio's data on size judgments if one assumes that category information is the verbal analogue of the nonverbal size information. More important, latency differences between pictures and words can be related to prediction equations derived from a single memory model and various dual memory models which make different assumptions about the location of category storage. In the section that follows, specific models are presented which represent these dual and single memory positions. The models presented and tested do not exhaust all possible models nor are they to be construed as formalizations of a particular theorist's position, although they may be consistent with such positions.

\section{Processing Parameters of Single and Dual Memory Models}

Each of the models to be presented share certain parameters or processing stages. These common parameters reflect encoding and output processes which are required irrespective of the number of internal memory stores postulated.

$a=$ picture encoding time, that is, the time for a picture to access its appropriate internal memory store.

$a^{\prime}=$ word encoding time, that is, the time for a word to access its appropriate internal memory store.

\section{$\mathrm{d}=$ decision time for the superordinate category match.}

$\mathrm{o}=$ output or response time.

The dual and single memory models differ witn respect to additional parameters which are required to describe processing in the semantic category judgment task. The only additional parameter required by a unitary model is the superordinate retrieval time within the single memory store.

$$
\mathrm{r}=\begin{aligned}
& \text { superordinate retrieval time in permanent } \\
& \text { memory. }
\end{aligned}
$$

The following are additional parameters required by the dual memory models to be discussed. The first set relate directly to the necessary transfer functions between multiple stores.

$t=$ transfer time from the nonverbal to the verbal symbolic system.

$t^{\prime}=$ transfer time from the verbal to the nonverbal symbolic system.

The second set relate to assumptions concerning where the superordinate category information is stored.

$\mathrm{r}_{\mathrm{NV}}=$ superordinate retrieval time if category information is stored in the nonverbal symbolic system, and/or

$\mathrm{r}_{\mathrm{V}}=$ superordinate retrieval time if category information is stored also (or only) in the verbal symbolic system.

The role of each of these parameters for making specific predictions about response latency for a given condition and differences in response latency between conditions is precisely described in the following section. Three general classes of models will be presented with two representing a dual memory position and the third representing the single memory position. Additional models will be considered in a final discussion section. All three model classes are represented in terms of additive serial processing stages. The models preser,ted and their associated condition and prediction equations can be converted into parallel processing representations without changing the major predictions. To avoid redundancy, only the serial processing representation has been employed.

\section{Dual Memory Model with Categorical Information in Both Systems}

The first dual memory model to be considered is one which assumes that category information may be simultaneously represented in both symbolic systems. The processing stages represented in Table 1 show that in this model both picture-picture and word-word category decisions require processing within only one of the symbolic systems. Prediction Equation la in Table 1 reveals that the latency difference between picture-picture and word-word conditions provides an estimate of processing time differences between the verbal and nonverbal systems. If word-word minus picture-picture latency is zero, then the combined access 
Table 1

Processing Stages, Condition and Prediction Equations for a Dual Memory Model with Categorical Representation in Both the Nonverbal and Verbal Symbolic Systems

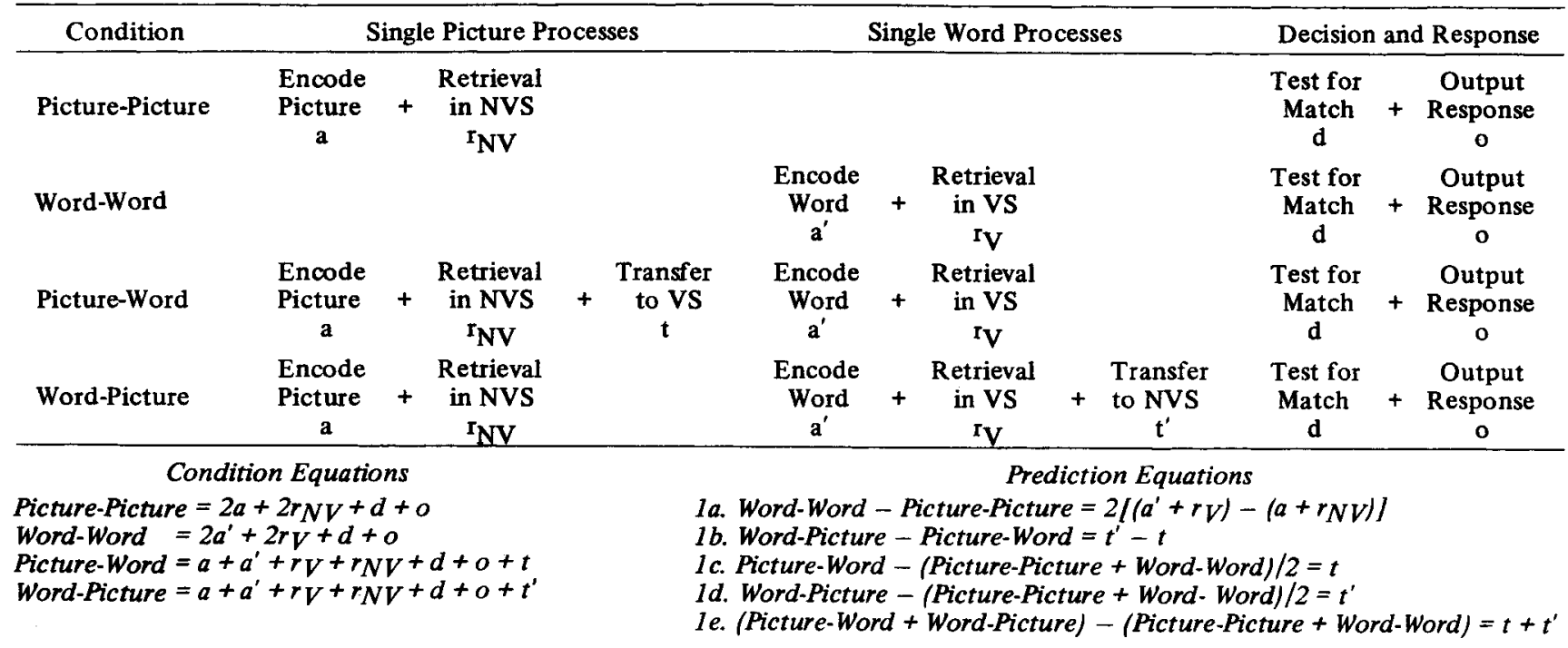

and retrieval time functions within each system are equivalent. If the direction of this difference is negative, then the combined access and retrieval time function is faster for words than for pictures. If the direction of this difference is positive, then the converse is true. It must be noted that this model does not permit separate estimates of the access and retrieval time parameters.

The processing stages represented in Table 1 also show that the picture-word and word-picture conditions require processing within both systems and the transfer of information between the two systems. The direction of information transfer is assumed to depend upon which system is activated first. In the picture-word condition the nonverbal system is activated first and thus information is transferred from the nonverbal to the verbal system with time $t$. The reverse set of operations is assumed to occur in the word-picture condition and transfer is from the verbal to the nonverbal system with time $t^{\prime}$. Prediction Equation $1 \mathrm{~b}$ in Table 1 shows that the latency difference between picture-word and word-picture conditions provides an estimate of the difference between $t$ and $t^{\prime}$, and according to the general dual memory position with $t<t^{\prime}$, this should be nonzero. The remaining three prediction equations (1c-e) relate the picture-word and word-picture conditions to the picture-picture and word-word conditions. These are important comparisons since they provide estimates of $t$ and $t^{\prime}$. These estimates should also be nonzero values. It is also important to note that these estimates do not depend upon the previously discussed relationship between the combined access and retrieval time function for each separate processing system. ${ }^{1}$

Although this is not represented in Table 1 , one might also assume that, regardless of which system is activated first, information is always transferred in one direction. ${ }^{2}$ Thus, in the picture-word and wordpicture conditions, transfer might always be from the nonverbal to the verbal system. Therefore, word-picture minus picture-word times should equal zero and Prediction Equations 1c, $1 \mathrm{~d}$, and le, respectively, estimate $t, t$, and $2 t$. If the direction of transfer is always from the verbal to the nonverbal system, then word-picture minus picture-word should again equal zero and Prediction Equations 1c, 1d, and 1e, respectively, estimate $t^{\prime}, t^{\prime}$, and $2 t^{\prime}$.

\section{Dual Memory Model with Categorical Information in the Verbal Symbolic System}

The alternative dual memory model to be considered assumes that semantic category information is represented within the verbal symbolic system. The processing stages represented in Table 2 show that in this model only the word-word condition involves the activation of a single system. Since the other three conditions involve pictures, both the nonverbal and verbal systems must be activated and information must always be transferred from the nonverbal to the verbal system. Prediction Equation 2a shows that word-word latency minus picture-picture latency is an estimate of the access time difference minus the transfer time $t$ which is required for information to flow from the nonverbal to verbal system. The following inferences follow directly from the difference between word-word and picture-picture latencies.

If $a^{\prime} \leqslant a$, then word-word - picture-picture is a negative value.

If $a^{\prime}>a$ and $\left(a^{\prime}-a\right) \leqslant t$, then word-word picture-picture is negative or zero.

If $a^{\prime} \gg \mathrm{a}$ and $\left(a^{\prime}-a\right)>t$, then word-word picture-picture is positive.

Thus, the only case in which Prediction Equation 2a can 
Table 2

Processing Stages, Condition and Prediction Equations for a Dual Memory Model with Categorical Representation in the Verbal Symbolic System

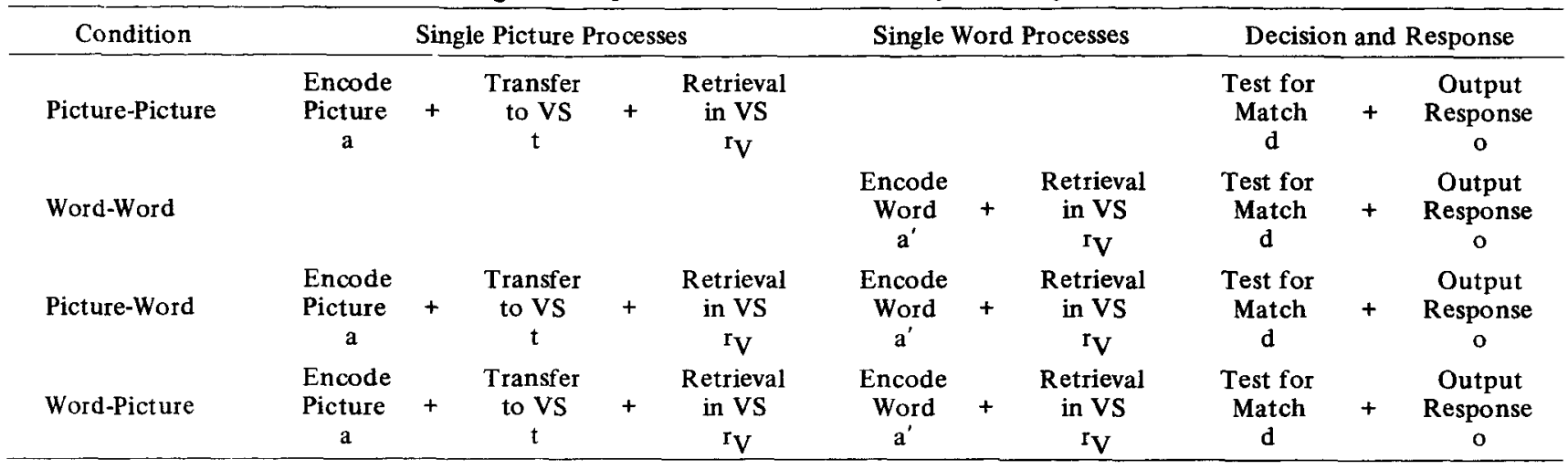

Condition Equations

Picture-Picture $=2 a+2 r V+d+o+2 t$

Word-Word $=2 a^{\prime}+2 r V+d+o$

Picture-Word $=a+a^{\prime}+2 r V+d+o+t$

Word-Picture $=a+a^{\prime}+2 r V+d+o+t$
Prediction Equations

2a. Word-Word - Picture-Picture $=2\left(a^{\prime}-a-t\right)$

2b. Word-Picture - Picture-Word $=0$

2c. Picture-Word - (Picture-Picture + Word-Word $) / 2=0$

2d. Word-Picture - (Picture-Picture + Word-Word) $/ 2=0$

2e. $($ Picture-Word + Word-Picture $)-($ Picture-Picture + Word - Word $)=0$ assume a positive value is when the difference in access time for pictorial vs. verbal stimuli is large relative to the transfer time $t$.

The remaining prediction equations (2b-e) all yield zero values and this is in contrast to the values predicted by the initial dual memory model with categoric information stored in both symbolic systems. Thus, these equations form a basis for selecting the best fitting dual memory model with respect to assumptions concerning the storage of categorical information.

\section{Unitary Memory Model}

The unitary memory model presented in Table 3 assumes that in all cases words and pictures access a single common memory store. Prediction Equation 3a thus indicates that the latency difference between word-word and picture-picture conditions is an estimate of the access time differences for pictorial and verbal stimuli. This equation is also applicable to Paivio's size judgment data, and therefore it predicts that the latency difference observed in the present semantic category task should approximate $187 \mathrm{msec}$. Thus, a latency difference of this value would support such a unitary memory model and seriously question the preceding dual memory model, while a latency difference which is negative for Equation 3a and approximates -187 would be interpreted as questioning the unitary memory model and supporting the preceding dual memory model. Equations $3 \mathrm{~b}-\mathrm{e}$ all predict values of zero and form the basis of a comparison with the

Table 3

Processing Stages, Condition and Prediction Equations for a Unitary Memory Model

\begin{tabular}{|c|c|c|c|c|c|c|c|c|c|}
\hline \multirow{2}{*}{$\frac{\text { Condition }}{\text { Picture-Picture }}$} & \multicolumn{3}{|c|}{ Single Picture Processes } & \multicolumn{3}{|c|}{ Single Word Processes } & \multicolumn{3}{|c|}{ Decision and Response } \\
\hline & $\begin{array}{c}\text { Encode } \\
\text { Picture } \\
\text { a }\end{array}$ & + & $\begin{array}{c}\text { Retrieval } \\
\text { in US } \\
r\end{array}$ & & & & $\begin{array}{c}\text { Test for } \\
\text { Match } \\
\text { d }\end{array}$ & + & $\begin{array}{c}\text { Output } \\
\text { Response } \\
\mathbf{o}\end{array}$ \\
\hline Word-Word & & & & $\begin{array}{c}\text { Encode } \\
\text { Word } \\
a^{\prime}\end{array}$ & + & $\begin{array}{c}\text { Retrieval } \\
\text { in US } \\
\mathbf{r}\end{array}$ & $\begin{array}{l}\text { Test for } \\
\text { Match } \\
\text { d }\end{array}$ & + & $\begin{array}{c}\text { Output } \\
\text { Response } \\
\text { o }\end{array}$ \\
\hline Picture-Word & $\begin{array}{c}\text { Encode } \\
\text { Picture } \\
\text { a }\end{array}$ & + & $\begin{array}{c}\text { Retrieval } \\
\text { in US } \\
r\end{array}$ & $\begin{array}{c}\text { Encode } \\
\text { Word } \\
a^{\prime}\end{array}$ & + & $\begin{array}{c}\text { Retrieval } \\
\text { in US } \\
r\end{array}$ & $\begin{array}{l}\text { Test for } \\
\text { Match } \\
\text { d }\end{array}$ & + & $\begin{array}{c}\text { Output } \\
\text { Response } \\
\text { o }\end{array}$ \\
\hline Word-Picture & $\begin{array}{c}\text { Encode } \\
\text { Picture } \\
\text { a }\end{array}$ & + & $\begin{array}{c}\text { Retrieval } \\
\text { in US } \\
\mathbf{r}\end{array}$ & $\begin{array}{c}\text { Encode } \\
\text { Word } \\
a^{\prime}\end{array}$ & + & $\begin{array}{c}\text { Retrieval } \\
\text { in US } \\
\text { r }\end{array}$ & $\begin{array}{l}\text { Test for } \\
\text { Match } \\
\text { d }\end{array}$ & + & $\begin{array}{c}\text { Output } \\
\text { Response } \\
\text { o }\end{array}$ \\
\hline
\end{tabular}

\section{Condition Equations}

Picture-Picture $=2 a+2 r+d+o$

Word -Word $=2 a^{\prime}+2 r+d+o$

Picture-Word $=a+a^{\prime}+2 r+d+o$

Word-Picture $=a+a^{\prime}+2 r+d+o$
Prediction Equations

3a. Word-Word - Picture-Picture $=2\left(a^{\prime}-a\right)$

3b. Word-Picture - Picture-Word $=0$

3c. Picture-Word - (Picture-Picture + Word-Word $) / 2=0$

3d. Word-Picture - (Picture-Picture + Word-Word $) / 2=0$

3e. (Picture-Word + Word-Picture $)-($ Picture-Picture + Word-Word $)=0$ 
initial dual memory conceptualization. Finally, it must be noted that the predictions for this model are also consistent with those that can be derived from a dual memory model with category information stored in the nonverbal symbolic system. Further consideration of this dual memory model will be reserved for the final discussion.

\section{Subcategory and Individual Item Effects}

The present study also included a further manipulation designed to address the general issue of categorical representation in multiple memory systems or in a single system where the latter could be either a unitary permanent memory or the verbal symbolic system of a dual memory model. For those pairs representing a common semantic category (animals or articles of clothing), half involved common subcategorizations or groupings (e.g., duck-chicken), while the other half involved different subcategories (e.g., trout-goat). If the system which stores semantic category information, either the unitary permanent memory or the verbal sy-mbolic memory, is hierarchically or multidimensionally organized (e.g., Collins \& Quillian, 1969; Rumelhart \& Abrahamson, 1973), then decision times on pairs whose members are derived from common subcategories should be faster than on pairs whose members are derived from different subcategories. This effect should occur for all types of pair presentations: picture-picture, picture-word, word-picture, and word-word. In the case of the unitary memory model, this is because all four presentation modes involve access to and decisions within a single permanent memory. In the case of the dual memory model with information stored in the verbal symbolic system, this is because all four presentation modes require retrieval and decision within the same verbal symbolic system. Consequently, both the unitary memory model and the dual memory model with categorical information in the verbal symbolic system would predict a significant linear relationship between decision times for individual word-word and picture-picture pairs.

The dual memory model with categorical information stored in both systems does not yield the same set of predictions. Paivio (1974) has argued that the verbal and nonverbal symbolic systems are not isomorphic in their underlying structure or organization. Thus, there need be no necessary linear relationship between individual picture-picture and word-word pairs since each pair type would involve access to and decision within a differently organized system. A strong linear relationship and a subcategory effect for both picture-picture and word-word pairs would imply an isomorphism in structure or organization. Even if one were to allow for the linear relationship and the subcategory effect in these conditions, there may be no subcategory effect in the picture-word and wordpicture conditions. Because information about category membership is being retrieved in each system and transferred, retrieval and transfer may occur at the level of the highest superordinate. As an example, in the pair trout-goat, retrieval and transfer of the superordinate fish would not lead to a match with the superordinates for goat unless retrieval and transfer occurred at the level animal. Thus, the demands of the task to match at a given level of abstraction could require transfer of information between the two systems at that level.

\section{Summary}

Three models representing dual memory and single memory theories have been presented and discussed with respect to assumptions about the nature of storage of categorical information. The assumptions of these models have further been related to specific predictions about latency differences in a category matching task. The three model classes described in the preceding sections can be compared and contrasted in terms of several aspects of the data. The values obtained for Prediction Equations b-e provide a basis for determining whether categorical information is simultaneously represented in two memory systems or represented in only a single memory system, either the verbal symbolic system of a dual memory model or a unitary permanent memory system. The major criterion for acceptance of the dual storage position requires that all the values obtained for Equations be be significant positive values. Other less critical data bearing on this same issue include the presence of a linear relationship between individual picture-picture and word-word pair decision latencies and the presence of subcategory effects in all four presentation conditions.

Should the results fail to support the dual storage assumption, the value obtained for Prediction Equation a provides an indirect test of the viability of a unitary memory model as opposed to a dual memory model with categorical storage only. in the verbal symbolic system. If this is a significant positive value, then the unitary memory model will receive support. A value of zero or a significant negative value would provide support for the dual memory position.

\section{EXPERIMENT 1}

\section{Method}

Design. The present design involves three between-subjects factors and three within-subjects factors. The three betweensubjects factors were: replication (two levels referring to each of the different stimulus sets), condition sequence (four levels referring to the Latin square counterbalancing), and sex. The within-subjects factors were presentation condition (picturepicture, picture-word, word-picture, and word-word), type of judgment (same or different), and individual item pairs (this represented a factor nested within both judgment type and replication). Additionally, the "same" judgment pairs could be further divided into the factors of semantic category (animals and clothing) and subcategory similarity (similar and dissimilar). When the latter breakdown is considered, the individual pairs factor becomes nested within subcategory similarity within semantic category within replication.

Materials and procedure. The stimuli were constructed from 20 examples of each of two broad semantic categories, 
animals and articles of clothing, and were chosen on the basis of ease of representation as simple line drawings. The 20 items selected within each category were divided into 10 pairs of items which represented common subcategories within the larger categories. In the animals category, these subcategories represented pairs of mammals (including farm and domestic), birds, fish, and insects. For the clothing category, the subcategories represented articles worn on the same part of the body (e.g., head, chest, legs, feet, and hands). These 20 pairs of similar items were then used to construct two stimulus sets, one for each replication of the design. Half of the similar pairings in each major semantic category were used in one stimulus set and the other half were used in the second stimulus set. The dissimilar subcategory pairings within each stimulus set were constructed from the remaining pairs by crossing subcategories within the remaining set of items. Thus, the items within categories which represented similar subcategory pairings in one replication constituted the dissimilar subcategory pairings in the other replication and vice versa.

Different judgment pairs were constructed by crossing items from the two major semantic categories (animals and clothing) with a total of 20 "different" judgment pairs in each stimulus set. Each replication contained a unique set of "different" judgment pairs and a unique set of "same" judgment pairs. Across both same judgment and different judgment pairs and replications, the left-right position of a particular item was counterbalanced. Thus, the total stimulus materials consisted of 40 unique pairs which represented different semantic categories and 40 unique pairs which represented the same semantic category. Within the latter there were 20 pairs ( 10 each within animals and clothing) which represented similar subcategories within the larger category and 20 pairs which represented dissimilar subcategories within the larger category.

Each subject was tested on only one of the replications, thus requiring him/her to make 20 same judgments and 20 different judgments for each presentation condition. Identical pairings were used within subjects across the four presentation conditions. The four presentation conditions were picturepicture, picture-word, word-picture, and word-word, where the condition labels refer to the mode of visual presentation of each member of the stimulus pair and the left-right ordering. All stimulus presentations involved the simultaneous presentation of both members of the stimulus pair. The pictorial stimuli were simple black and white line drawings approximately $3 \times 5$ in., and the visually presented verbal stimuli were typed in lowercase letters approximately 1.5 in. high.

Each subject was tested for 160 reaction time trials, which were divided into four blocks of 40 trials each. Each block of 40 trials represented one of the four presentation conditions. Within each block of 40 trials, there were five blocks of eight trials each with four same and four different judgment pairs in each block. The four same judgment pairs represented one example each of similar and dissimilar subcategory pairs within each of the two major semantic categories. Four different orders of condition sequence were used, and these represented a Latin square counterbalancing procedure whereby each presentation condition preceded and followed every other condition once across the four sequences.

Before beginning the experimental session, the subject was given 20 to 40 practice trials on a same-different reaction time task involving the presentation of individual pairs of letters. This pretraining was to familiarize the subject with the apparatus and to establish a stable response time. The subject was then instructed about the subsequent task and the basis for a samedifferent decision in terms of the two specific semantic categories to be considered. No mention was made of similar and dissimilar subcategories within the two major semantic categories. The subjects were requested to respond as quickly as possible without making errors. To control for the possibility of a speed-accuracy tradeoff, a $95 \%$ minimum accuracy rate was required of each subject in each presentation condition. All subjects met or exceeded this criterion of accuracy.
Apparatus. All stimuli were presented via carousel projector and back-projected onto a translucent glass screen. The latter was approximately $12 \times 10 \mathrm{in}$. and located approximately 18 in. from the subject. The stimuli were projected at eye level and horizontally centered on the screen for each left-right member of each pair. At the base of the screen was a $6 \times 10$ in. response panel containing two response buttons labeled "same" and "different." The response button for same was always on the left and different always on the right. The subject rested the preferred hand midway between the two response buttons prior to each new stimulus presentation. Response latency was measured to the nearest millisecond by a Hunter Klockcounter. The timing circuit was initiated by the projector beam activating a photocell and the subject's response terminating the timing interval. The interstimulus interval was approximately $10 \mathrm{sec}$, the amount of time required to record response latency and accuracy and to reset the apparatus.

Subjects. The subjects were 32 University of Pittsburgh undergraduates participating to fulfill a course requirement. All subjects were tested in individual sessions approximately $45 \mathrm{~min}$ in length and were randomly assigned in their order of appearance to one of the between-subjects conditions for a particular sex.

\section{Results}

The reaction times of each subject on each individual pair of items were subjected to two separate analyses of variance. The minimum significance level adopted for both analyses was $1 \%$, due to the number of factors and observations involved and the resultant sensitivity of the analyses. In the first analysis, replications, condition sequence, and sex were between-subjects factors and presentation condition and type of judgment were within-subjects factors. In addition, in this analysis individual pairs were analyzed as a nested factor. Across replications, there were 40 unique pairs representing same judgments and 40 unique pairs representing different judgments.

The main effect of presentation condition was highly significant $[F(3,48)=27.35, p<.001]$, and this is shown in Figure 2. The overall means were $715 \mathrm{msec}$ for picture-picture pairs, $810 \mathrm{msec}$ for picture-word pairs, $851 \mathrm{msec}$ for word-picture pairs, and $900 \mathrm{msec}$ for word-word pairs. The significant Same-Different by Presentation Condition interaction $[F(3,48)=4.33$,

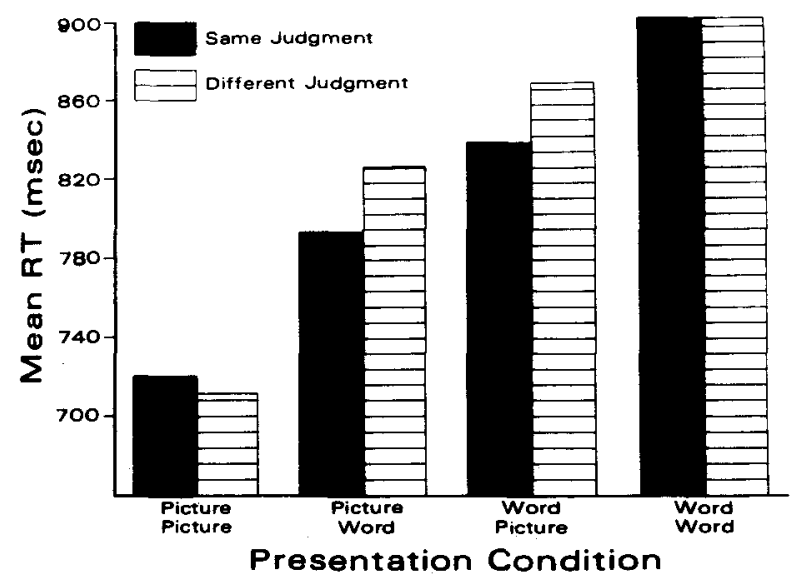

Figure 2. Mean reaction time as a function of type of judgment and presentation condition. 
Table 4

Observed Values for Prediction Equations a-e as a Function of Same, Different, and Combined Judgments

\begin{tabular}{lrrr}
\hline & Same & Different & Overall \\
\hline a. Word-Word - Picture-Picture & $180^{*}$ & $191^{*}$ & $185^{*}$ \\
b. Word-Picture - Picture-Word & 40 & 42 & 41 \\
c. Picture-Word - (Picture-Picture + Word-Word) $/ 2$ & -16 & 21 & 3 \\
d. Word-Picture - (Picture-Picture + Word-Word) $/ 2$ & 24 & 43 & 4 \\
e. (Picture-Word + Word-Picture) - (Picture-Picture + Word-Word) & 8 & 84 \\
\hline
\end{tabular}

${ }^{*} p<.01 ;$ all other values $p>.05$.

$\mathrm{p}<.01]$ is also shown in Figure 2. Generally, the overall pattern of differences among picture-picture, pictureword, word-picture, and word-word conditions was identical for both same $(720,794,834$, and $900 \mathrm{msec}$, respectively) and different judgments $(709,825,867$, and $900 \mathrm{msec}$, respectively). The significant interaction effect was due primarily to faster reaction times (32 msec) to same than to different picture-word and word-picture pairs and somewhat slower reaction times $(5 \mathrm{msec})$ to same than to different picture-picture and word-word pairs. No other main effects or interactions were significant.

Separate Scheffé contrasts $(\alpha=.01, \beta=.99)$ were performed to test the condition differences obtained from Prediction Equations a-e. These data are summarized in Table 4 for the overall values as well as the separate same and different judgment items. As can be seen in Table 4, the values obtained for Equations b-e were generally small and in one case negative. The latter results call into question the first dual memory model with categorical storage in both symbolic systems. Since Equations b-e all provide nonsignificant estimates of transfer time functions, this model appears untenable. The value for Equation a is highly significant and its positive value is supportive of a unitary memory model as opposed to a dual memory model with categorical storage in the verbal symbolic system.

As a further test of the generality of the conditions effect, the presentation conditions variance was tested against the Presentation Condition by Nested Pairs interaction $[F(3,228)=103.66, p<.001]$. The latter highly significant effect is reflected by the fact that for 79 of 80 pairs, the picture-picture mean was less than the corresponding word-word mean. The latter $F$ value and that obtained for presentation condition when tested against the Presentation Condition by Subjects variance were used to calculate min $\mathrm{F}^{\prime}$ (Clark, 1973 ), which was also significant $\left[\min F^{\prime}(3,76)=21.64\right.$, $\mathrm{p}<.001]$. This indicates that the presentation condition effect is generalizable to both other subject and item samples.

Subcategory similarity. To examine the effect of subcategory similarity, a second analysis was performed on the reaction times for each subject on only the same judgment pairs (subcategory differences do not exist as a factor for different judgment pairs). These reaction times were subjected to an analysis in which replications, condition sequence, and sex were again between-subjects factors, and presentation condition, category type, and subcategory similarity were within-subjects factors. Individual pairs were again analyzed as a nested factor.

As expected from the first analysis, the main effect of presentation condition was highly significant $[F(3,48)=23.91, p<.001]$, and this can be seen in Figure 3. The mean reaction times for similar and dissimilar subcategory pairs were 689 and $750 \mathrm{msec}$, respectively, for picture-picture pairs, 878 and $922 \mathrm{msec}$ for word-word pairs, 762 and $825 \mathrm{msec}$ for picture-word pairs, and 782 and $886 \mathrm{msec}$ for word-picture pairs. Figure 3 also shows the main effect of subcategory similarity $[F(1,16)=29.22, p<.001]$. The mean reaction time to pairs in which the two items belonged to a common subcategory ( $778 \mathrm{msec})$ was faster than to pairs in which the items belonged to different subcategories $(846 \mathrm{msec})$. No other main effects or interactions were significant.

Separate Scheffé contrasts were again performed to test Prediction Equations a-e, and these values are shown in Table 5 . The values are separately reported for the similar and dissimilar pairs. As can be seen in Table 5, the values obtained for Equations b-e were again small, and in four of eight cases negative. Thus,

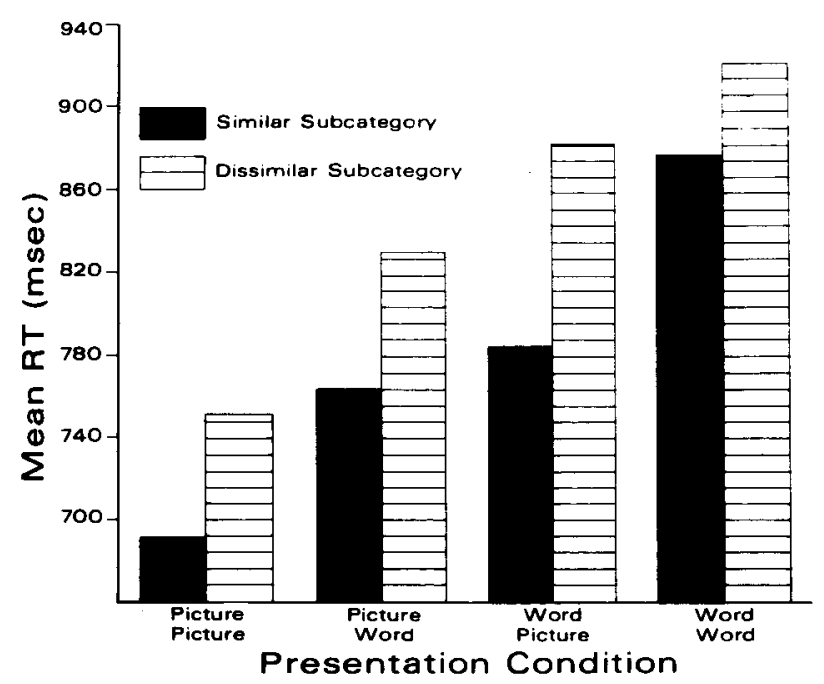

Figure 3. Mean reaction time to same judgment pairs as a function of similarity of subcategory and presentation condition. 
Table 5

Observed Values for Prediction Equations a-e as a Function of Similar and Dissimilar Same Judgment Pairs

\begin{tabular}{lrr}
\hline & Similar & Dissimilar \\
a. Word-Word - Picture-Picture & $189 *$ & $172 *$ \\
b. Word-Picture - Picture-Word & 20 & 61 \\
c. Picture-Word - (Picture-Picture + Word-Word)/2 & -22 & -11 \\
d. Word-Picture - (Picture-Picture + Word-Word)/2 & -2 & 50 \\
e. (Picture-Word + Word-Picture) - (Picture-Picture + Word-Word) & -23 & 39 \\
\hline
\end{tabular}

${ }^{*} p<.01 ;$ all other values $p>.05$.

as noted before, the small, sometimes negative, and nonsignificant estimates of transfer time functions call into question the first dual memory model with categorical storage in both symbolic systems. The existence of a subcategory similarity effect in all presentation conditions also argues against such a model and in favor of the storage of category information in a single system. As expected from the overall analysis, Prediction Equation a yields highly significant values for both similar and dissimilar pairs, and these values again can be interpreted as supportive of a unitary memory model.

As in the first analysis, the presentation condition effect was tested against the Presentation Condition by Nested Pairs interaction $[F(3,96)=45.17, p<.001]$. The $F^{\prime}$ test was also significant $\left[\min F^{\prime}(3,98)=15.63\right.$, $\mathrm{p}<.001]$. The subcategory similarity effect was also tested against the nested pairs variance, and this was also significant $[F(1,32)=17.64, p<.001]$. The $\mathrm{F}^{\prime}$ test for subcategory similarity was also significant $\left[\min F^{\prime}(1,48)=11.00, \quad p<.005\right]$. Thus, both the presentation condition and subcategory similarity effects are generalizable to both other subject and item samples.

Regression analysis. As argued in the introduction, if semantic decisions for picture-picture and word-word pairs are being made in a single memory system, then a linear relationship between picture-picture and wordword decision times for individual pairs should be found. The consistency of the subcategory similarity effect across presentation conditions suggests that such a relationship may exist. To determine more precisely the nature of this relationship, mean picture-picture and word-word reaction times on individual item pairs were subjected to regression analyses separately for same and different judgment pairs. The results of these analyses are shown in Figure 4 . The product-moment correlations between picture-picture and word-word reaction times were $.64(38 \mathrm{df}, \mathrm{p}<.01)$ for same judgment pairs and .58 (38 df, $\mathrm{p}<.01$ ) for different judgment pairs. The least squares regression equations are also shown in Figure 4 and the slope and intercept values were similar for both same and different judgment pairs. Each slope value was tested for the difference from 1.00 and neither comparison was signficant $[\operatorname{ts}(38)<1.47, \mathrm{ps}>.05]$. The existence of such comparable linear functions for both the same and different judgment pairs further argues that category information is stored in a single system, rather than in two separate systems which have different underlying organizational structures.

It could be argued that the relationship between word-word and picture-picture latencies is an artifact of the general frequency with which the individual items occur in normal language or experience. In order to test this possibility, simple and multiple correlations were computed with word-word latency as the criterion and printed frequency and picture-picture latency as the predictors. The same judgment pairs showed that printed frequency alone accounted for $22 \%$ of the variance in word-word latency, and the inclusion of picture-picture latency as an additional predictor significantly increased the variance accounted for to a value of $48 \%$. The different judgment pairs showed that printed frequency alone accounted for only $1 \%$ of the variance in word-word latency, and the inclusion of picture-picture latency as an additional predictor significantly increased the variance accounted for to a value of $35 \%$. We do not have any compelling explanation for the reduction in variance accounted for in the different judgment pairs. Nevertheless, the analyses clearly show that the relationship between word-word and picture-picture latencies was not an artifact of frequency.

\section{Discussion}

Dual storage vs. single storage. The results of the present experiment will be considered first with respect

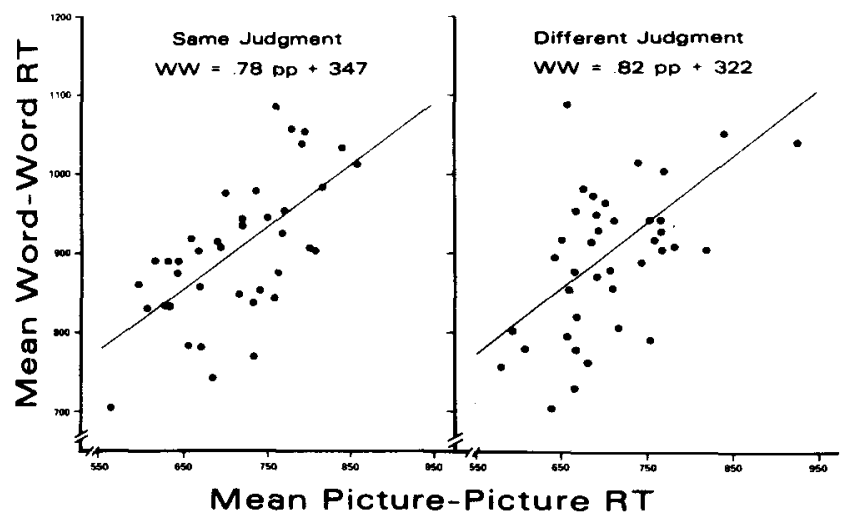

Figure 4. Scatter plots of individual pair reaction time shown separately for same and different judgment pairs. Also shown are least-squares regression equations and best-fit lines. 
to differentiating between the dual memory model with categorical representation in both systems vs. the single and dual memory models with categorical representation in a single memory system. As outlined in the introduction, there are several data components which address this issue. The most critical comparison between these two positions rests on the values obtained for Prediction Equations b-e, particularly Equation e. Although the values in Tables 4 and 5 for these transfer parameters were based upon many observations (from 640 to 5,120 ), none approached significance. In an absolute sense, the obtained estimates were small and in several cases negative in value. In contrast, all of these values were predicted to be zero by both the unitary memory model and the dual memory model with categorical storage in the verbal symbolic system. Thus, the latter two models and the general concept of categorical storage in a single system seem to be supported.

Other support for the concept of storage in a single system was provided by the significant linear relationship between decision times on individual picture. picture and word-word pairs. Such a relationship implies that the same memory system was mediating the individual pair decisions. If the decisions about individual pairs were being made in separate systems, then the relationship implies a strong similarity in the structure or organization of both systems.

The final aspect of the data which is consistent with storage in a single system was the occurrence of a subcategory similarity effect in all four presentation conditions. As was the case for the linear relationship between individual pair decision latencies, such a result implies that the same memory structure was mediating the superordinate decisions regardless of mode of presentation. If this is a hierarchically organized semantic system (e.g., Collins \& Quillian, 1969), it would be expected that similarity of subcategory should lead to faster decision times, since the point of intersection of the two concepts being compared should occur at a subcategory node below the level of the more superordinate category node. This effect was observed in the present data and the difference in reaction times for similar and dissimilar subcategory pairs $(68 \mathrm{msec})$ closely approximated the slope of the function obtained by Collins and Quillian (1969). However, it should be noted that the present results are also consistent with an explanation based on associative frequency between the elements within a pair (e.g., Conrad, 1972) or a multidimensional space representation of conceptual categories (e.g., Rumelhart \& Abrahamson, 1973). What is of major importance here, however, is that this subcategorization effect was consistently observed across all four presentation conditions.

Our findings that estimates of transfer time are nonsignificant, that there is a linear relationship between individual word-word and picture-picture pair decision latencies, and that subcategory similarity effects exist in all conditions provide a strong argument against the dual memory model with categorical storage in both the verbal and nonverbal symbolic systems. All aspects of the data converge on and are consistent with models which posit categorical representation in a single memory system. The question which was addressed by the next experiment is whether this single system is the verbal symbolic memory of the dual memory model or the abstract permanent memory of the single memory model.

\section{EXPERIMENT 2}

The purpose of the second experiment was to provide a direct test of the relationship between picture and word decision latencies in semantic category and size judgment tasks. Such a comparison allows one to discriminate between the unitary memory model and the dual memory model with categorical storage in the verbal symbolic system. The semantic category task involved the presentation of single pictures or words, with the subject required to decide if the stimulus was an animal or an object. These two broad categories were used because of limitations on item selection within the Paivio (1975) size norms. The size judgment task was similar in format to the category judgment task, except that the subject was required to decide if the stimulus presented was large or small. The stimuli consisted of four sets of items: large and small animals and large and small objects.

The unitary and dual memory models yield different predictions for the relationship between picture and word conditions across these two decision tasks. In the case of the dual memory model, it is assumed that category information is represented in the verbal symbolic system, whereas size information is represented in the nonverbal symbolic system (e.g., Paivio, 1974, 1975). The word-picture difference in the size judgment task is thus an estimate of $a^{\prime}-a+t^{\prime}$. The word-picture difference in the category judgment task is an estimate of $a^{\prime}-a-t$. Thus, the word-picture difference in the size judgment task should be larger than the difference in the category judgment task. By subtracting the second difference from the first, an estimate of $t^{\prime}+t$ is obtained. Clearly, this should be a significant nonzero value. In contrast, the unitary memory model predicts identity for the relationship between word-picture differences in both tasks.

\section{Method}

Design. The present design included two between-subjects factors and four within-subjects factors. The between-subjects factors were task order (size judgment preceding or following category judgment) and presentation sequence (four sequences representing different orders of item presentation). The withinsubjects factors were judgment task (category vs. size), stimulus type (pictures vs. words), item category (animal or object), and item size (large or small).

Materials and procedure. The stimulus material consisted of 40 items selected from Paivio's (1975) norms for items 
scaled on the dimension of size. There were 10 items each from the four stimulus subsets representing large animals, small animals, large objects, and sinall objects. The mean size for each of these four stimulus subsets was 6.11 for large animals (range 5.45-7.37), 2.93 for small animals (range 2.27-3.61), 5.66 for large objects (range 5.02-6.88), and 2.48 for small objects (range 2.02-3.18).

Four sequences of stimulus presentation were constructed. Each sequence consisted of a 40-trial block involving the presentation of each item only once. Within each 40 -trial block, half of the items were presented as pictures and the other half as words. Each 40-trial block was also arranged such that successive sets of four items contained one item from each of the four stimulus subsets, and these items were randomized within each set of four. Individual items were repeated across blocks with an average lag of 39 intervening items. The four sequences of item presentation formed the basis for a Latin square counterbalancing procedure.

The pictures were simple black and white line drawings approximately $3 \times 5$ in., and the visually presented verbal stimuli were typed in uppercase letters approximately 1.5 in. high. The picture stimuli were all drawn such that they occupied approximately the same amount of area, and thus no unique cues were provided for size relationships. The apparatus used in Experiment 1 was also used for the presentation of stimuli in this experiment.

Each subject was tested for 160 reaction time trials which were divided into two 80-trial blocks. Each 80-trial block represented one of the two judgment tasks. Before beginning each 80-trial block, the subject was given practice trials with picture and word stimuli to familiarize the subject with the apparatus and the type of decision that was required. In the size judgment task, the subject was given a point of reference for the small-large decision. The subject was told to consider items to be small if they could be readily held by a normal adult. The subjects had no difficulty in making this small-large dichotomy.

Subjects. The subjects were 24 University of Pittsburgh undergraduates participating to fulfill a course requirement. All subjects were tested in individual sessions approximately $45 \mathrm{~min}$ in length and were randomly assigned to conditions in their order of appearance.

\section{Results}

The overall level of accuracy in the category judgment task was high for both pictures $(99.3 \%)$ and words $(98.1 \%)$. As might be expected, the size judgment task yielded a somewhat lower level of correct item assignments for both pictures $(94.0 \%)$ and words (94.2\%). The correct picture and word decision latencies for each subject were averaged within each of the four stimulus subsets and these average values were then

Table 6

Mean Reaction Time in Experiment 2 as a Function of Judgment Task, Stimulus Type, Item Category, and Item Size

\begin{tabular}{lccccc}
\hline & \multicolumn{2}{c}{ Animal } & & \multicolumn{2}{c}{ Object } \\
\cline { 2 - 4 } \cline { 5 - 5 } & Large & Small & Large & Small \\
\hline Picture & 600 & 592 & 638 & 631 \\
Word & 674 & 709 & 705 & 662 \\
& & \multicolumn{4}{c}{ Category Judgment } \\
Picture & 717 & 762 & 732 & 757 \\
Word & 767 & 770 & 832 & 742 \\
\hline
\end{tabular}

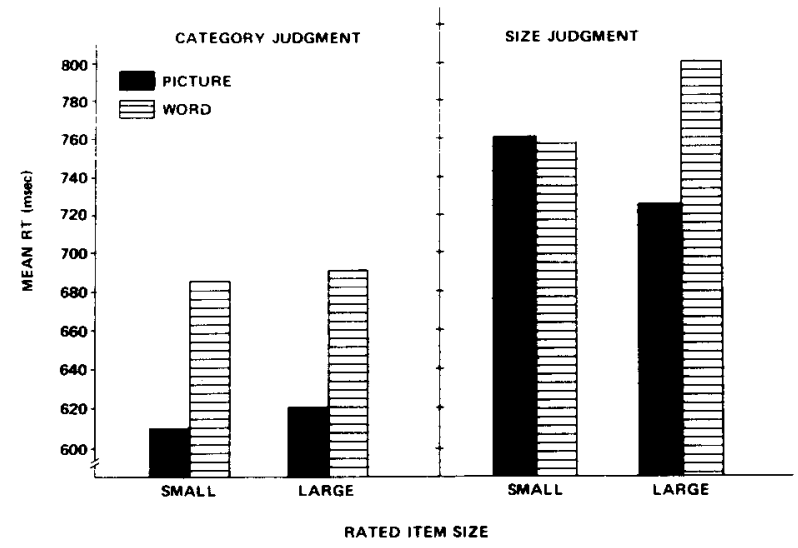

Figure 5. Mean reaction time as a function of stimulus type, item size, and judgment condition.

analyzed. Table 6 presents the mean latencies for each of the experimental factors.

The analysis of correct decision latencies showed that category judgments $(651 \mathrm{msec})$ were faster than size judgments $(760 \mathrm{msec})[\mathrm{F}(1,22)=79.8, \mathrm{p}<.001]$. The animal stimuli yielded faster latencies $(699 \mathrm{msec})$ than the object stimuli $(712 \mathrm{msec})[F(1,22)=7.31$, $\mathrm{p}<.05]$. There also was an interaction between item size and item category $[F(1,22)=12.57, p<.01]$ : Small animals yielded a longer average latency than large animals $(19 \mathrm{msec})$, while the opposite item size effect occurred for the object stimuli $(-29 \mathrm{msec})$.

The major effects of importance concerned the picture vs. word factor. Pictures yielded faster decision latencies $(679 \mathrm{msec})$ than words $(734 \mathrm{msec})$ $[F(1,22)=85.22, \quad p<.001]$. The Picture-Word by Judgment Task by Item Size interaction was highly significant $[F(1,22)=27.0, p<.001]$. This interaction is graphically presented in Figure 5. Pictures yielded faster decision latencies than words in the category judgment task (73 and $70 \mathrm{msec}$ differences for small and large items, respectively). In the size judgment task, this difference was found only for large items $(74 \mathrm{msec})$; there was no difference between pictures and words in size judgments of small items $(-3 \mathrm{msec})$.

The pattern shown in Figure 5 was also reflected in picture-word differences on individual items. Median latencies of individual items within the category judgment task showed picture decision latency faster than word decision latency for 38 of the 40 items. Median latencies of individual items within the size judgment task showed picture decision latency faster than word decision latency for 18 of the 20 large items, but only 8 of the 20 small items.

The median picture and word decision latencies on individual items were correlated for both the category and size judgment tasks. In the category task, separate correlations were obtained for the animal and object stimuli and the average correlation was $.45(\mathrm{p}<.005)$. 
In the size judgment task, separate correlations were obtained for the small and large stimuli and the average correlation was $.52(\mathrm{p}<.005)$. Thus, the correlations obtained for both types of judgment were similar in magnitude to those obtained in Experiment 1, and are consistent with the proposition that decisions are being made in a single memory system.

\section{Discussion}

The present results on picture-word differences in category decision latency clearly support the findings obtained in Experiment 1. In the first experiment, pairs of pictures yielded latencies $185 \mathrm{msec}$ faster than pairs of words. This value is an estimate of $2\left(a^{\prime}-a\right)$ in the unitary memory model or $2\left(a^{\prime}-a-t\right)$ in the dual memory model with category storage in the verbal system. In the present experiment, single pictures yielded latencies $72 \mathrm{msec}$ faster than single words. This value is assumed to estimate $a^{\prime}-a$ in the unitary model and $a^{\prime}-a-t$ in the dual memory model. The present results on size decision latency also showed a pattern consistent with the predictions of the unitary memory model as well as Paivio's $(1974,1975)$ data on relative size decisions. The unitary memory model predicts that picture-word differences in size decision latency should equal or approximate those obtained for category decision latency. This was clearly the case for size judgments on large animals and objects where pictures were $74 \mathrm{msec}$ faster than words.

No difference was obtained for size judgments on small animals and objects. This finding can be related to Paivio's $(1974,1975)$ finding on size congruency and incongruency. As noted earlier, in the task used by Paivio, the pairs of pictures or words were presented such that the two projected stimuli represented equal or unequal physical sizes. In the case of equal physical sizes, both items were either physically large or small and under these conditions the picture-word latency difference was $187 \mathrm{msec}$. In the case of unequal physical size, when there was congruence between the physical stimulus sizes and the relative size decision, the difference between picture and word pairs was $207 \mathrm{msec}$. However, in the case of incongruence between physical size and the relative size decision, the difference between picture and word pairs was reduced to $106 \mathrm{msec}$. The latter was entirely attributable to the effect of size incongruence on the picture pairs.

The present picture-word size judgment data on small animals and objects can be interpreted as reflecting a size incongruence for pictures similar to that obtained by Paivio. While only single stimuli were presented, all the picture stimuli were drawn to approximately equal size, and when projected, they were large relative to the total area of the projected visual display. Thus, small animals and objects when projected pictorially represented an incongruence between the relative projected size and the appropriate internal size information. In Paivio's data, the resolution of such an incongruence reduced the picture-word difference by
$100 \mathrm{msec}$ and in the present study the resolution of incongruence reduced the picture-word difference by 77 msec, yielding no difference between single pictures and words.

The present data appear to be consistent, then, with both the category data obtained in Experiment 1 and Paivio's size judgment data. The comparison of word-picture differences across the category and size judgment tasks provides a test of the viability of the unitary memory model as opposed to that of the dual memory model with category information in the verbal system and size information in the nonverbal system. In the dual memory model, subtracting the word-picture difference in the category task from the word-picture difference in the size task yields an estimate of $t^{\prime}+t$. If the times for size judgments on both small and large items are included, then the estimate of $t^{\prime}+t$ is $-36 \mathrm{msec}$. However, a more appropriate estimate is obtained by considering size judgments on only the large items. The latter estimate is $2.5 \mathrm{msec}$. Thus, the direct comparison of picture and word decision latencies across category and size tasks yields a nonsignificant transfer estimate. This clearly indicates that the unitary memory model provides a more consistent explanation of picture-word processing differences across both perceptual and semantic decision tasks.

\section{GENERAL DISCUSSION}

The results from Experiments 1 and 2 allow us to reject a dual memory model with categorical information simultaneously represented in both symbolic systems. Results from Experiment 2 allow us to reject a model with category information represented in the verbal symbolic system and size information represented in the nonverbal system. The data dictate a model(s) in which both size and category information are represented in the same symbolic system. Furthermore, any model must be able to account for the fact that pictures have faster access to this knowledge system than do words. Three models fit these requirements. It is possible that both size and category information could be represented in the verbal symbolic system of a dual memory model. Applying this model to the data results in an estimate that the access time differences between pictures and words is large relative to any transfer time necessary for the processing of pictures.

A second model consistent with the data assumes that both size and category information are represented in the nonverbal symbolic system. In this case, the verbal system primarily acts as an acoustic-phonemic decoder and makes relatively little contribution to any deeper level of analysis (e.g., categorical).

The third model is the unitary memory model previously described, which assumes that there is a single underlying system and different access routes to that system. Data presently available do not permit selection among these three types of models. Consequently, 
decisions about the underlying nature of memory must be based on other grounds. Based simply on parsimony, one could argue that the unitary memory model is most appropriate insofar as it only requires the assumption of access time differences and makes no assumptions about transfer time operations. We acknowledge that the three models are functionally equivalent and thus make no further attempt to differentiate among them. Instead, we will consider what may be the source of the process time difference and what justification beyond the present data can be provided for this difference. There are at least two possible alternatives relative to the source of this difference: (1) Analogous processing stages are required for pictorial and verbal input, but the time required for each stage is less for pictorial input, or (2) pictorial inputs require fewer processing transformations than verbal inputs before semantic processing occurs. The latter alternative seems more defensible since it can be related to the assumption that the acoustic-phonemicarticulatory coding of verbal input has no analogous counterpart for pictorial processing. Evidence by Dhawan and Pellegrino (in press) in a probe-memory task has shown that picture memory shows semantic interference and reduced acoustic interference throughout the list, whereas word memory shows semantic interference only for initial list items and acoustic interference for terminal list items. This suggests that the more recent verbal items go through an acoustic processing stage (see also Shulman, 1971), whereas pictorial processing involves no such stage and there is more direct access to semantic processing. Data provided by Bartram (1974) for picture naming latency and Potter (1975) for picture recognition at rapid rates of presentation have also been interpreted in terms of rapid picture access to an abstract memory system.

Another argument for the differential access rates and the elimination of processing stages with pictorial stimuli can be based upon the degree of correspondence between pictorial and verbal representations and their external referents (see also Rosch, 1975). Pictures or line drawings are representations of real world objects and use the same features as their external referents. Words, however, are arbitrarily derived codes (i.e., re-representations) with no necessary feature overlap with their external referents. Words thus contain features which must be processed before the semantic features of the referent can be processed. Additionally, developmental research by Hochberg and Brooks (1962) has demonstrated picture identification in an infant with little or no previous training or experience with pictures. This is in contrast to the slow development of verbal processing skills, particularly in the case of visually presented verbal material. If the difference in access times reflects differential processing ability, this suggests that for highly skilled verbal processors differences between access times for pictorial and verbal input for high-familiarity concepts may be reduced. Rader (Note 1) has shown that graduate students with high verbal aptitude scores (above 700) fail to show significant picture-picture vs. word-word differences in semantic decision times for a task similar to the present one. Conversely, one would expect the differences in access time to increase for less skilled verbal processors. Rosinski, Pellegrino, and Siegel (1977) have shown that in the identical semantic decision task, the difference in access times was 399 and $242 \mathrm{msec}$ for second and fifth graders, respectively.

Given that there are differences in processing time for pictorial and verbal input, the final issue to be considered is the implication of this difference with respect to data often cited as support for the dual memory position.

A major source of evidence taken as support for this theory is the consistent superiority of pictorial over verbal input in long-term retention tasks (Paivio, 1971). However, these results do not require a dual memory model and can be explained in terms of speed and level of semantic processing. Since retention depends upon the depth or breadth of postperceptual processing where semantic levels lead to superior retention relative to orthographic or acoustic levels, any factors such as processing constraints or instructions (e.g., imagery or verbal mediation) which increase the depth or breadth of processing within a single system will produce superior retention. To the extent that pictures involve a more rapid access to semantic levels and the possibility for more extensive processing at this level relative to their verbal counterparts, then picture retention should be superior for equal and restricted rates of presentation (see also Nelson \& Reed, 1976).

The absence of a negative recency effect with pictorial as compared to verbal stimuli (Madigan, McCabe, \& Itatani, 1972) also is consistent with a level of processing difference between pictures and words. The occurrence of negative recency has been related to a lower level of processing for verbal stimuli in the recency portion of the list (Maskarinec \& Brown, 1974; Watkins \& Watkins, 1974). The lack of negative recency for pictures can be interpreted as evidence that the deeper semantic processing levels have been reached even for pictures at the end of the list. This is also consistent with the previously discussed semantic interference data of Dhawan and Pellegrino (in press).

This particular example illustrates that, regardless of whether one wishes to embrace a unitary or dual memory model, the present data clearly suggest that further research on pictorial vs. verbal input must focus on access and process time differences rather than on a hypothesized number of representations and their locations.

\section{REFERENCE NOTE}

1. Rader, N. L. Developmental changes in getting meaning from written words. Paper presented at the meeting of the 
Society for Research in Child Development, Denver, April 1975.

\section{REFERENCES}

ANDERSON, J. R., \& Bower, G. H. Human associative memory. Washington, D.C: V. H. Winston, 1973.

Atkinson, R. C., \& Shiffrin, R. M. Human memory: A proposed system and its control processes. In $K$. W. Spence \& J. T. Spence (Eds.), The psychology of learning and motivation (Vol. 2). New York: Academic Press, 1968.

Bartram, D. J. The role of visual and semantic codes in object naming. Cognitive Psychology, 1974, 6, 325-356.

Chase. W. G., \& Clark, H. H. Mental operations in the comparison of sentences and pictures. In $L$. W. Gregg (Ed.), Cognition in learning and memory. New York: Wiley, 1972.

CLARK, H. H. The language-as-fixed-effect fallacy: A critique of language statistics in psychological research. Journal of Verbal Learning and Verbal Behavior, 1973, 12. 335-359.

Collins, A. M.. \& Quillian, M. R. Retrieval time from semantic memory. Journal of Verbal Learning and Verbal Behavior, 1969, 8, 240-247.

Conrad, C. Cognitive economy in semantic memory. Journal of Experimental Psychology, 1972, 92, 149-154.

Craik, F.' I., \& Lockhart, R. S. Levels of processing: A framework for memory research. Journal of Verbal Learming and Verbal Behavior, 1972, 11, 671-684.

Crowder, R. G.. \& Morton, J. Precategorical acoustic storage (PAS). Perception \& Psychophysics, 1969, 5, 365-373.

Dhawan, M., \& Pellegrino, J. W. Acoustic and semantic interference effects in words and pictures. Memory \& Cognition, 1977, 5, 340-346.

Hochberg, J. E., \& Brooks, V. Pictorial recognition as an unlearned ability: A study of one child's performance. American Journal of Psychology, 1962, 75, 624-628.

Kroll, N. E., Parkinson, S. R., \& Parks, T. E. Sensory and active storage of compound visual and auditory stimuli. Journal of Experimental Psychology, 1972, 95, 32-38.

Madigan, S., McCabe, L., \& Itatan, E. Immediate and delayed recall of words and pictures. Canadian Journal of Psychology, 1972, 26, 407-414.

Maskarinec, A. S., \&.Brown, S. C. Positive and negative recency effects in free recall learning. Journal of Verbal Learning and Verbal Behavior, 1974, 16, 328-334.

NeIsser, U. Cognitive psychology. New York: AppletonCentury-Crofts, 1967.

Nelson, D. L., \& REED, U. S. On the nature of pictorial encoding: A levels-of-processing analysis. Joumal of Experimental Psychology: Human Learning and Memory, 1976. 2, 49-57.

PaIvio, A. Imagery and verbal processes. New York: Holt, Rinehart. \& Winston, 1971.

Palvio. A. Images, propositions, and knowledge. Research Bulletin No. 309, University of Western Ontario, 1974.
Parvio, A. Perceptual comparisons through the mind's eye. Memory \& Cognition, 1975, 3, 635-647.

Pellegruno, J. W., Siegel, A. W., \& Dhawan, M. Shortterm retention of pictures and words: Evidence for dual coding systems. Joumal of Experimental Psychology: Human Learning and Memory, 1975, 104, 95-102.

Pellegrino, J. W., Siegel, A. W., \& Dhawan, M. Shortterm retention of pictures and words as a function of type of distraction and length of delay interval. Memory \& Cognition, 1976, 4, 11-15.

Penney, C. G. Modality effects in short-term verbal memory. Psychological Bulletin. 1975, 11, 68-84.

Potter. M. C. Meaning in visual search. Science, 1975 , 187, 965-966.

Pylyshyn, $Z$. W. What the mind's eye tells the mind's brain: A critique of mental imagery. Psychological Bulletin, 1973, 80, 1.24.

Rosch, E. Cognitive representations of semantic categories. Journal of Experimental Psychology: General, 1975, 1. 192-233.

Rosinski, R. R., Pellegrino, J. W., \& Siegel, A. W. Developmental changes in the semantic processing of pictures and words. Joumal of Experimental Child Psychology, 1977, 23, 282-291.

Rumelhart, D. E., \& ABrayamson, A. A. A model for analogical reasoning. Cognitive Psychology, 1973 5. 1-28.

Shulman, H. G. Similarity effects in short-term memory. Psychological Bulletin, 1971, 75, 399-415.

SPERLING, G. The information available in brief visual presentations. Psychological Monographs, 1960 , 74(Whole No. 498).

Watkins. M. J., \& Watkins, O. C. Processing of recency items for free recall. Journal of Experimental Psychology, $1974,102,488-493$

WICKELGREN, W. A. The long and the short of memory. Psychological Bulletin, 1973, 80. 425-438.

\section{NOTES}

1. An alternative version of this model could also assume different decision time parameters $d_{y}$ and $d_{n v}$. This model predicts that Equation 1a will equal $2\left(a^{\prime}+r_{v}\right)-\left(a+r_{n v}\right)+d_{v}-d_{n v}$. Thus, the word-word - picture-picture difference will reflect access, retrieval, and decision time differences between the verbal and nonverbal systems. The values for Equations $1 \mathrm{~b}-\mathrm{d}$ will no longer be estimates of just transfer times. However, Equation le will still estimate $t+t^{\prime}$

2. This same argument also applies to a processing strategy in picture-word and word-picture conditions, whereby the subject always attends to the picture (or word) stimulus first.

(Received for publication January 20, 1977; revision accepted April 26, 1977.) 